\title{
Congratulations! Dual Graphs are Now Orientated!
}

\author{
Darshan Batavia ${ }^{1}$, Walter G. Kropatsch ${ }^{1}$, Rocio M. Casablanca ${ }^{2}$, and Rocio \\ Gonzalez-Diaz ${ }^{2}$ \\ 1 TU Wien, Pattern Recognition and Image Processing Group 193/03, Austria \\ \{darshan,krw\}@prip.tuwien.ac.at \\ 2 Applied Math I, University of Seville, Spain \\ $\{$ rociomc, rogodi\}@us.es
}

\begin{abstract}
A digital image can be perceived as a 2.5D surface consisting of pixel coordinates and the intensity of pixel as height of the point in the surface. Such surfaces can be efficiently represented by the pair of dual plane graphs: neighborhood (primal) graph and its dual. By defining orientation of edges in the primal graph and use of Local Binary Patters (LBPs), we can categorize the vertices corresponding to the pixel into critical (maximum, minimum, saddle) or slope points. Basic operation of contraction and removal of edges in primal graph result in configuration of graphs with different combinations of critical and non-critical points. The faces of graph resemble a slope region after restoration of the continuous surface by successive monotone cubic interpolation. In this paper, we define orientation of edges in the dual graph such that it remains consistent with the primal graph. Further we deliver the necessary and sufficient conditions for merging of two adjacent slope regions.
\end{abstract}

\section{Introduction}

Configuration of such critical points and slope lines of a surface in term's of earth topography were discussed in 211]. Nackmann Lee in [10] investigated the configurations of critical points of a Morse function of two variables with it's graphical representation. Moving a century ahead, in [3], authors used the neighborhood graph and explained the use of Local Binary Patterns (LBPs) in predicting the critical (maxima, minima and saddle) points and the slope points in digital images. By performing contraction and removal operations on edges, they formed a stack of graphs called graph pyramid. Cerman et al 4 . provide a practical application of multi-resolution image segmentation using graph pyramids. Similar approaches are used by Wei in 12 where a hierarchical structure similar to graph pyramid were constructed by using superpixels. The literature except [3] does not consider the topological aspect of the surfaces which are covered by the papers mentioned in the following paragraph.

In 667. Edelsbrunner et al. propose an algorithm of constructing a hierarchy of increasingly coarse Morse-Smale complexes to decompose a piecewise linear 2D-manifold with all its critical points being distinct. In our previous research 
work 899, we further generalize this concept beyond Morse-Smale complex and present a new hierarchy of increasingly coarse complexes decomposing $2 \mathrm{D}$ continuous surfaces denoted as slope complexes.

We also discussed properties of monotonic paths and provided a formula to count the number of slope regions at the top level of the pyramid with all its critical points being distinct. Our main aim was to preserve the critical points of the surface at the top level of the pyramid and to connect them with the minimum number of slope regions calculated using Euler's formula [5, Theorem 4.2.7].

In the past, the graph pyramids 3 are build on the top of the pair of dual graphs. The contraction of edges generates self-loops and multiple edges. The empty self-loops and multiple edges can be removed and simplified. This simplifications are controlled by the dual graphs. These dual graphs were not oriented and also were not used to capture the non topological properties of the graph. The main contribution of this paper is to provide orientation to the dual graph. A first step in this direction was done in 9 but here we provide it's interpretation in the image context and give properties of the oriented dual graphs to provide a reduction technique to meet our aims.

The paper is organized as follows: We start with the basic definitions in the field of images from the topological point of view. In section 3, we define a concept to orient the dual graphs and the consistency of LBP categories. We also introduce a technique to orient the monotonic paths which contains level curves in the primal graphs. Section 4 provides necessary and sufficient conditions for both primal and dual graph to merge two slope regions. In section 5, we show some experimental results for multi-resolution image segmentation. We end the paper with a note of what is attained from the paper and the possible extensions.

\section{Orienting the Primal Graph}

This section provides necessary definitions that form the basis of the further document. A discrete 2D image $P$ where the intensity of a pixel $p$ denoted by $g(p)$, can be represented as a neighborhood graph $G=(V, E)$ also referred to as primal graph. Every pixel $p$ in the image $P$ corresponds to a vertex $v \in V$ with gray value (g-value) $g(v):=g(p)$. Vertex $v$ is connected to it's four adjacent vertices by edges $e \in E$.

The dual of primal graph $G$ is denoted by $\bar{G}=(\bar{V}, \bar{E})$, where $\bar{V}$ being the vertex set of $\bar{G}$ which is associated with the faces of $G$, while $\bar{E}$ is the edge set of $\bar{G}$ which corresponds to the borders separating the faces of $G$. In other words, there is an edge $\bar{e}$ in the dual graph $\bar{G}$ for every edge $e$ in primal graph $G$ as mentioned in [5, Section 4.6]. There is a one-to-one correspondence between the edges of $G$ and $\bar{G}$ so as the faces of $G$ and vertices of $\bar{G}$. By performing the contraction and removal operations successively on the graph $G$, we obtain a stack (pyramid) of successively reduced plan $\oint^{3}$ graphs $\left(G_{k}, \overline{G_{k}}\right), k \in[1,2, \ldots, n]$.

${ }_{3}$ There is a topological and a combinatorial isomorphism between $G$ and $\bar{G}$ and it is a unique pair of graphs embedded in a surface [5. p. 70-80] 
The base level of this pyramid is denoted as $G=G_{0}$. A Contraction operation in $G_{k}, 0 \leq k \leq n-1$ corresponds to removal in $\overline{G_{k}}$ and a removal operation in $G_{k}$ (merging of two faces) corresponds to contraction of two vertices in $\overline{G_{k}}$.

Definition 1. The orientation of an edge $(v, w) \in E$ in the primal graph $G=(V, E)$ is directed from vertex $v \in V$ to vertex $w \in V$ iff $g(v)>g(w)$, all the other edges are not oriented.

Orientation of edges can now be used to categorize the vertex $v \in V$ into critical (maximum, minimum, saddle) or slope point.

Definition 2. A vertex $v \in V$ is a local local maximum $\oplus$ if all the edges incident to $v$ are oriented outwards.

Definition 3. A vertex $v \in V$ is a local minimum $\ominus$ if all the edges incident to $v$ are oriented inwards.

Definition 4. A vertex $v \in V$ is a saddle point $\otimes$ if there are more than two changes in the orientation of edges when traversed circularly (clockwise or counter-clockwise direction).

Definition 5. A vertex $v \in V$ is a slope point if there are exactly two changes in the orientation of edges when traversed circularly (clockwise or counter-clockwise direction).

Categorizing a vertex using orientation of edges incident to it is equivalent to that of LBP code. The LBP value of an outward oriented edges are encoded as 1 and inward orientated edges are encoded as 0 . The LBP code of a vertex is formed by concatenating LBP values of the incident edges in clockwise or counter-clock wise direction. The LBP code of a maximum will consist of 1 only while the LBP code of a minimum will consist of 0 only. The LBP code of slope points will have exactly 2 bit switches and saddles will have more than 2 bit switches. By use of orientated edges, we avoid the calculation of derivatives and eigen-values of the Hessian matrix to categorize a vertex.

Definition 6. A path $\pi\left(v_{1}, v_{r}\right)=\left(V_{\pi}, E_{\pi}\right)$ is a non empty sub-graph of $G=$ $(V, E)$, where $V_{\pi}=\left\{v_{1}, \ldots, v_{r}\right\} \subset V$ and $E_{\pi}=\left\{\left(v_{1}, v_{2}\right), \ldots,\left(v_{r-1}, v_{r}\right)\right\} \subset E$.

A path $\pi\left(v_{1}, v_{r}\right)$ is a monotonic path if all the oriented edges $\left(v_{i}, v_{i+1}\right), i \in$ $[1, r-1]$ have a same orientation.

Remark 1. All the oriented edges on a monotonic path have the same orientation consequently defining the orientation of a monotonic path. Observe that if $g\left(v_{i}\right)=g\left(v_{i+1}\right), \forall i \in[1, r-1]$ is called a level curve and it is a special case of monotonic paths.

A monotonic path $\pi\left(v_{1}, v_{r}\right)$ can be further extended by adding an edge oriented in the same direction as the direction of monotonic path $\pi\left(v_{1}, v_{r}\right)$. A monotonic path which cannot be further extended is called a maximal monotonic path. The end points of a maximal monotonic path will always be a local maximum and a local minimum. 


\subsection{Contracting plateaus}

A connected sub-graph having the same g-value for all the vertices is referred to as a plateau region where every pair of vertices $v, w \in V$ of the sub-graph satisfies $g(v)=g(w)$. The LBP encoding (see next subsection) is performed after contraction of all the edges in the plateau regions where each plateau region collapses either to a single vertex in the best case or a set of self-loops attached to a vertex, surrounding every hole in the plateau region [8].

Nevertheless, the vertices on the boundary of the image need to be treated differently. First, the case where the plateau region is connected to the boundary as shown by the shaded region in the left images of Fig. 11(a),(b), needs to be treated specially. To preserve the topology, we first perform contraction on the vertices corresponding to the pixel on the boundary of the image.

As a result we get the vertices through which the border is connected to the plateau region, and then collapse the remaining part of the plateau region into a level curve as shown in the right images of Fig. 1(a),(b). Simultaneously, it also explains the reason to perform the operations on border independently and prior to the edges encapsulated by the border.

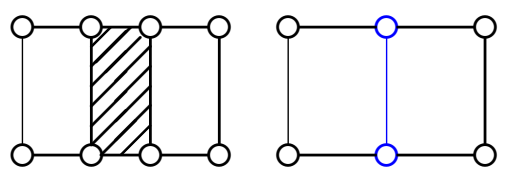

(a)

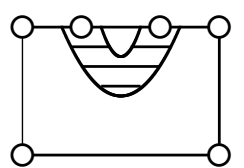

(b)

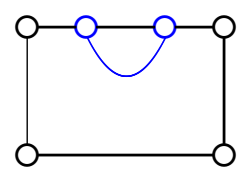

Fig. 1. Plateau regions connected to the boundary.

\section{Orientation of dual graphs}

In this section, we introduce a method to orient the edges of the dual graph corresponding to the oriented edges of the primal graph and explore it's properties to help merging of faces in the primal graph. Maintaining generality, $\bar{e}$ in $\overline{G_{h}}$ is normal to the tangent at any point on the edge $e$ in $G_{h}$.

Definition 7 (Orientation of edges in dual graph). The orientation of an edge $\bar{e}$ in $\bar{G}$ is from $\overline{v_{r}}$ to $\overline{v_{l}}$, where $\overline{v_{r}}$ and $\overline{v_{l}}$ are the vertices in $\bar{G}$ corresponding to the faces on the right and the left side of the respective edge $e$ in $G$ while walking in the direction of orientation of $e$.

Remark 2. The condition where the LBP code of $\bar{v}$ in $\bar{G}$ consists of only $0^{*}$ or only $1^{*}$ can never exist as it corresponds to a cyclic sub-graph in $G_{k}$ and it contradicts the orientation of edges initially defined for the primal graph. Nevertheless, directed cycles in the dual graph can appear and they surround extrema in the primal.

Presence of level curves in $G_{k}$ may complicate the orientation of the corresponding edge in $\overline{G_{k}}$ which we address later in this document. 


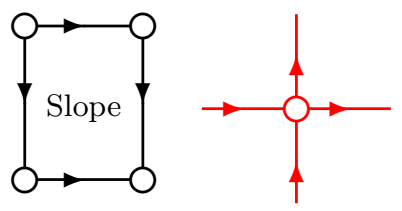

(a)

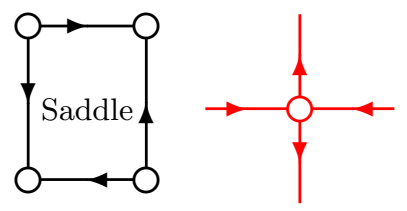

(b)

Fig. 2. Orientated dual graph for slope and saddle regions in primal graph.

Definition 8 (Slope region). A face in $G_{k}$ is a slope region iff the corresponding vertex $\bar{v}$ in $\overline{G_{k}}$ is a slope point.

Imagine the primal graph $G_{0}$ as a terrain with height corresponding to the g-value of the vertex, then a face in $G_{0}$ will be a slope region or a saddle region 4 in the terrain. Def. 7 remains consistent to this concept and gives a matching LBP category of a vertex in dual as shown in Fig. 2

Lemma 1 ([9]). The boundary of a slope region $S$ in the primal graph $G$ is either composed of exactly two monotonic paths connecting two extrema or it is a level curve.

Lemma 2. [1, Lemma 1]After contracting plateaus and adding dummy saddle points inside non-well composed configurations, all the vertices in the dual graph are slope points.

\section{Merging of two slope regions}

This section starts with basic requirements for merging of two slope regions. Then we provide the prototype of two adjacent slope regions sharing a common boundary. Finally we enumerate all the possible configurations of two slope regions deduced from the prototype and provide the constructive statements which are the necessary conditions for merging of two slope regions.

Two slope regions sharing a common boundary can be merged together by removal of the common boundary which results to form a merged slope region. We do not constrain the number of vertices and edges on the boundary of the slope regions. Hence the boundaries of the slope regions are referred as paths which may contain more than one vertices and edges. From Lemma 1 and properties of monotonic paths, the boundary of a slope region consist of exactly one level curve or two monotonic paths connecting one maximum and one minimum 'with respect to the slope region'. Note that by the usage of term 'with respect to the slope region', we constrain the connections of the vertex with the interior and the boundary of the slope region. We do not consider the connections of the vertex with the remaining graph where it can be categorized into a different LBP category.

\footnotetext{
${ }^{4}$ Region with a non well-composed configuration which requires insertion of a saddle point [3].
} 
Merging of two slope regions may not necessarily result in a slope region. The merging of two slope regions can be done by checking whether the resulting merged region is a slope region or not.

Remark 3. Two adjacent slope regions in the primal graph can be merged iff the dual vertex corresponding to the merged slope region would be a slope point.

According to Lemma 1, the boundary of a slope region should be composed of two separate monotonic paths connecting the maximum and minimum of the slope region. The vertices at the end point of the common boundary between the two slope regions are already part of two monotonic paths, one from each slope region. To be a part of a monotonic path, these two vertices should either be an extremum or a slope point with respect to the monotonic path. If this condition is violated, it will contradict to the orientation of the monotonic path on the boundary of the resulting slope region. Now the proof of this boils down to demonstrate that the dual vertex corresponding to a face (in the primal graph) surrounded by exactly two monotonic paths is a slope point. Considering the circular permutation of the LBP codes of above mentioned dual vertex, we will have exactly two switches which as per Def. 5 is a slope point.

Let us consider sub-graphs of two slope regions $S_{1}$ and $S_{2}$ with their extrema $\oplus_{1}, \ominus_{1}, \oplus_{2}$ and $\ominus_{2}$ respectively. While formulating rules for merging two slope regions, the position of an extrema on the boundaries and it's connection with the common boundary are the main features to be considered. Besides extrema, the boundaries are composed of slope points with respect to the slope region. In this way we provide conditions which are independent of the number of edges and vertices on the boundary of the slope regions $S_{1}$ and $S_{2}$.

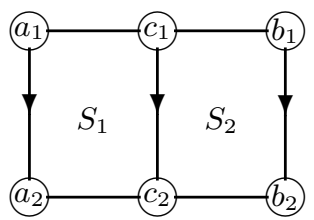

(a)

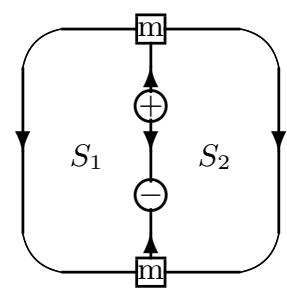

(b)

Fig. 3. Prototype for merging of two slope regions.

Fig. 3 a shows the prototype of two slope regions with a common boundary $\pi\left(c_{1}, c_{2}\right)$ between them. The paths connecting the vertices in the prototype may consist of any number of vertices and edges completing the respective connection. The orientation of paths $\pi\left(a_{1}, a_{2}\right), \pi\left(b_{1}, b_{2}\right)$ and $\pi\left(c_{1}, c_{2}\right)$ is predefined by assuming that $\oplus_{1} \in\left\{a_{1}, c_{1}\right\}, \oplus_{2} \in\left\{b_{1}, c_{1}\right\}, \ominus_{1} \in\left\{a_{2}, c_{2}\right\}$ and $\ominus_{2} \in\left\{b_{2}, c_{2}\right\}$ positions. The theory remains the same if the positions of $\oplus_{1}$ and $\oplus_{2}$ are interchanged with $\ominus_{1}$ and $\ominus_{2}$ thereby reversing the orientation (flipping) of respective 
edges 5 Moreover, all the combinations where the orientation of path $\pi\left(a_{1}, a_{2}\right)$ and $\pi\left(b_{1}, b_{2}\right)$ are opposite, for example $\oplus_{1}$ at $a_{1}$ and $\oplus_{2}$ at $b_{2}$ will not be valid because it contradicts the orientation of path $\pi\left(c_{1}, c_{2}\right)$ unless it is a level curve (which is taken into account in Case 5). By putting restrictions on positions, we reduce the number of possible combinations to $2^{4}=16$ configurations, which can be further reduced (by interchanging $S_{1}$ and $S_{2}$ ) for investigative purpose and still keeping the rules general.

The removal of a path $\pi\left(c_{1}, c_{2}\right)$ with $n$ edges consists of following two steps:

1. Contract $(n-1)$ edges as a result of which the path will be composed of only 1 edge connecting the end points.

2. Perform the removal operation on the remaining edge.

Following are enumerations in which the two slope regions $S_{1}$ and $S_{2}$ can be merged such that the resulting slope region will still obey Def. 8 and preconditions of Lemma 1. Preserving the condition, the number of different configurations which can be generated by interchanging labels $S_{1}$ and $S_{2}$ are mentioned in the brackets after each condition.

Case 1: We start with the most simple case of Fig. 4 a, where $S_{1}$ and $S_{2}$ share the same extrema on the common boundary, i.e. $\oplus_{1}=\oplus_{2}$ at $c_{1}$ and $\ominus_{1}=\ominus_{2}$ at $c_{2}$. In this case, remaining paths are directed from $c_{1}$ to $c_{2}$, one through $a_{1}$ and $a_{2}$, and the other through $b_{1}$ and $b_{2}$ [2 combinations].

Case 2: If the common boundary is composed of one extremum from $S_{1}$ and one extremum from $S_{2}$ as shown in Fig. $4 \mathrm{~b}$. [2 combinations.]

Case 3: If both of the end points of the common boundary contains extrema from a single slope region irrespective of the position of extrema from other slope region as shown in Fig. 5 a . For example: $\oplus_{1}$ at $a_{1}$ and $\ominus_{1}$ at $a_{2}$ respectively while $\oplus_{2}$ and $\ominus_{2}$ contribute to the end points of the common boundary. [6 combinations.]

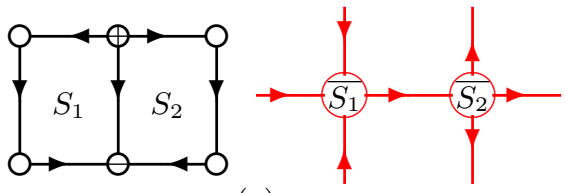

(a)

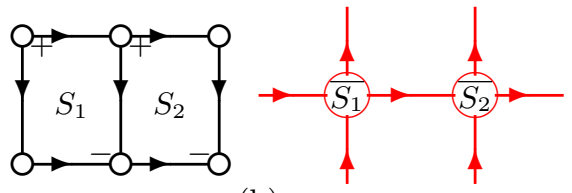

(b)

Fig. 4. Slope regions contributing at-least one extremum to the common boundary.

Case 4: None of the extrema contribute to the common boundary, but both extrema of one slope region are connected to the common boundary through a level curve as shown in Fig. 5b. The blue vectors besides the graph shows the orientation of the monotonic path which consist of level curves.[1 combination.] Case 5: If one extremum of a slope region contributes to the end point of the common boundary and the other is connected to the common boundary through

\footnotetext{
${ }^{5}$ This configuration can be achieved by switching positions of $\oplus_{i}$ and $\ominus_{i}$ in the previously mentioned configuration.
} 
a level curve irrespective of the position of extrema of the other slope region. For example refer to Fig. 55,d. The dashed line in the figure refers to the level curve and the blue vectors besides the graphs shows the orientation of the monotonic path. [6 combinations.]

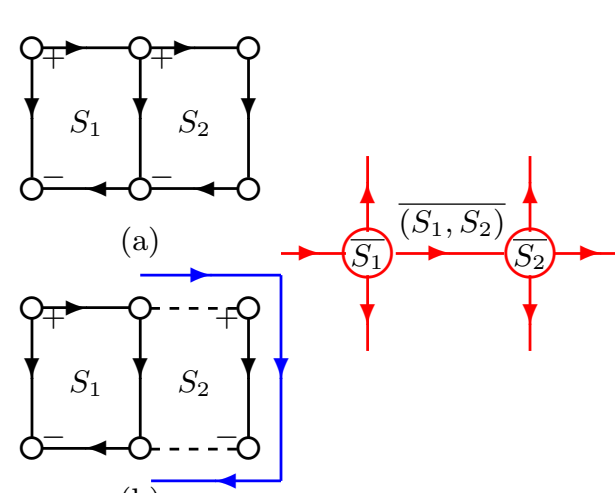

(b)

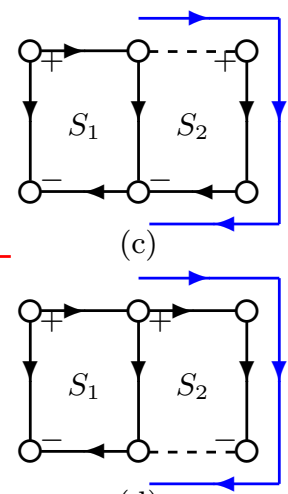

(d)

Fig. 5. At-least one extremum of a slope region is connected to the common boundary through a level curve.

In all the above cases, the common boundary path $\pi\left(c_{1}, c_{2}\right)$ has orientation in a single direction. We also take into consideration the cases where the common boundary have orientations of edges in different direction like shown in Fig. 3b, where the vertex $m$ is a slope point with respect to the slope regions. In its local neighborhood, $m$ can be a slope point or a saddle point. Note: This case is counted under the condition where both the slope regions share common extrema and hence the slope regions can be merged. There may be cases where only one of the two common slope points $m$ is present and the monotonic paths are directly connected to an extremum. Removal of path $\pi(\oplus, \ominus)$ in Fig. 3p will result in pending edges connecting $m$ and the extrema in the primal graph. Corresponding to this pending edge, there will a self-loop surrounding a single vertex in the dual graph.

Proposition 1. Any two slope regions $S_{1}$ and $S_{2}$ sharing a common boundary can be merged together if they follow one of the following condition:

1. The common boundary is composed of at least one extremum of $S_{2}$ and another extremum is either on the common boundary or is connected to the common boundary through a level curve.

2. The common boundary is composed of one extremum from each slope region.

For all the cases in Fig. 4 $4 \mathrm{~b}$ and Fig. 5 , the edges incident to the dual vertex $\overline{S_{1}}$ have the same orientation as the dual vertex $\overline{S_{2}}$ when traversed in a circular (clockwise or counter clockwise) order. Similar observations can be made in the corresponding primal graphs. 
In other words, both of the vertices in the dual exhibit the same LBP code after eliminating redundancy of bits when traversed in same direction. The vertex in Fig. 6 is a slope point formed as a result of the contraction operation on the dual graph in Fig. 5 . Similarly we get a slope point after contracting the dual edge connecting the two dual vertices in Fig. 4b.

Few examples can be viewed in Fig. 7 where the extrema are connected to the common boundary without a level curve or the orientation of the level curve

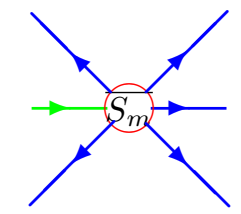

Fig. 6. Result after contraction of edge $\overline{\left(S_{1}, S_{2}\right)}$ in Fig 5 is reversed. We observe that the vertices in the dual graph have different LBP codes when traversed in the same direction.

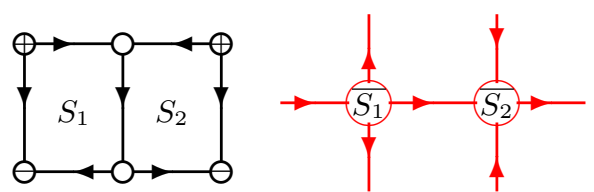

(a)

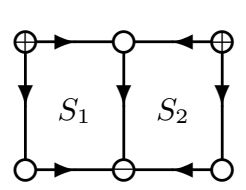

(b)

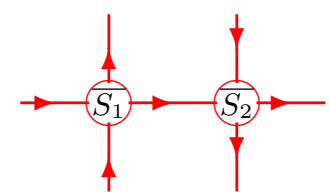

Fig. 7. Configurations of slope regions that cannot be merged and their oriented dual graphs.

Proposition 2. The contraction of the dual edge $\bar{e}$ connecting two slope points $\overline{v_{1}}$ and $\overline{v_{2}}$ in dual graph $\bar{G}$ will result in a single slope point iff the edges incident on the vertices $\overline{v_{1}}$ and $\overline{v_{2}}$ exhibit the same orientation ( the same LBP code) between the bit switches when traversed in same order and direction.

Proposition 2 provides sufficient conditions for contraction of edge $\bar{e}$ in $\bar{G}$ connecting two slope points such that the resulting dual vertex is also a slope point. This contraction is equivalent to the removal of the corresponding edge $e$ in $G$ such that the resulting region is a slope region.

\subsection{Orientation of level curves shared by multiple monotonic paths}

Technically level curves follow the surface along the same height. Hence they are not oriented. The corresponding (level) paths can be concatenated with adjacent monotonic paths if all the involved monotonic paths have the same orientation. They then form a combined monotonic path in which the level paths inherit their orientation from the orientation of the combined monotonic path. This may lead to inconsistencies if the same level path or a sub-sequence of it is simultaneously concatenated with a monotonic path of the opposite orientation. In such cases preference will be given to the orientation of the level path which is involved in the merging of slopes. The priority of merging slopes may depend on higher objectives like making slope regions of the global extrema as large as possible. 


\section{Conclusion}

In this paper, we represent a surface by a pair of dual plane graphs and provide a novel solution of collapsing the plateau region which basically is the collection of degenerated surface points. Then we categorize the vertices into maximum, minimum, slope or saddle depending on the orientation of the incident edges and avoiding the calculations of differentiation. We also define the orientation of the edges in the dual graph and show that the LBP category of the dual vertex is consistent with the corresponding face in the primal graph. Then we give the necessary and sufficient conditions for merging of two adjacent faces in the primal graph of a well composed sampled surface such that the merged face is a slope region. Finally we offer the sufficient conditions for the resulting dual vertex to be a slope point produced after contracting the respective dual edge.

\section{References}

1. Batavia, D., Kropatsch, W.G., Gonzalez-Diaz, R., Casblanca, R.M.: Counting slope regions in surface graphs. In: Computer Vision Winter Workshop. (2019) 5

2. Cayley, A.: Xl. on contour and slope lines. The London, Edinburgh, and Dublin Philosophical Magazine and Journal of Science 18(120), 264-268 (1859) 1

3. Cerman, M., Gonzalez-Diaz, R., Kropatsch, W.G.: LBP and Irregular Graph Pyramids. In: Petkov, N., Azzopardi, G. (eds.) Proceedings of the CAIP2015 (2015) 1. 2,5

4. Cerman, M., Janusch, I., Gonzalez-Diaz, R., Kropatsch, W.G.: Topology-based image segmentation using lbp pyramids. Machine Vision and Applications 27(8), 1161-1174 (2016) 1

5. Diestel, R.: Graph theory. 1997. Grad. Texts in Math (1997) 2

6. Edelsbrunner, H., Harer, J., Natarajan, V., Pascucci, V.: Morse-smale complexes for piecewise linear 3-manifolds. In: Proceedings of the nineteenth annual symposium on Computational geometry. pp. 361-370. ACM (2003) 1

7. Edelsbrunner, H., Harer, J., Zomorodian, A.: Hierarchical morse-smale complexes for piecewise linear 2-manifolds. Discrete and computational Geometry 30(1), 87107 (2003) 1

8. Kropatsch, W.G., Casablanca, R.M., Batavia, D., Gonzalez-Diaz, R.: Computing and reducing slope complexes. In: Computational Topology In Image Context. Springer (2019) 24

9. Kropatsch, W.G., Casablanca, R.M., Batavia, D., Gonzalez-Diaz, R.: On the space between critical points. In: Discrete Geometry for Computer Imagery. Springer (2019) 2,5

10. Lee, R.N.: Two-dimensional critical point configuration graphs. IEEE Transactions on Pattern Analysis and Machine Intelligence 4, 442-450 (1984) 1

11. Maxwell, J.C.: L. on hills and dales: To the editors of the philosophical magazine and journal. The London, Edinburgh, and Dublin Philosophical Magazine and Journal of Science 40(269), 421-427 (1870) 1

12. Wei, X., Yang, Q., Gong, Y., Ahuja, N., Yang, M.H.: Superpixel hierarchy. IEEE Transactions on Image Processing 27(10), 4838-4849 (2018) 1 